\title{
A NOTE ON THE SHIFT ON THE CANTOR SET
}

\author{
ROBBERT FOKKINK
}

(Communicated by James E. West)

\begin{abstract}
Let $O(x)$ be the orbit of $x$ under a homeomorphism $h$ on a metric space $X$. It is shown that this motion can be found in the shift on the Cantor set; i.e. there is an embedding $j: O(x) \rightarrow\{0,1\}^{Z}$ such that $\sigma \circ j=j \circ h$.
\end{abstract}

Let $\sigma:\{0,1\}^{Z} \rightarrow\{0,1\}^{Z}$ be the shift on the Cantor set defined by $\sigma\left(\left(x_{n}\right)_{n}\right)$ $=\left(x_{n+1}\right)_{n}$. In the thirties Marston Morse showed that there exists an element of $\{0,1\}^{Z}$ that is uniformly recurrent under $\sigma$. This element is now generally known as the Morse sequence; see $[\mathrm{MH}]$ or $[\mathrm{F}]$. The existence of such elements in $\{0,1\}^{Z}$ was not evident to the earliest investigators of symbolic dynamics. It is the object of this paper to show that the cascade $\left(\{0,1\}^{Z}, \sigma\right)$ contains copies of all possible orbits of cascades. We prove the following theorem:

Theorem. Let $h: X \rightarrow X$ be a homeomorphism on a metric space $X$. For every $x \in X$ there is an embedding $j: O(x) \rightarrow\{0,1\}^{Z}$ such that $\sigma \circ j=j \circ h . O(x)$ is the orbit of $x$ defined as $O(x)=\left\{h^{n}(x) \mid n \in Z\right\}$.

As observed in [A], the possible topological types of $O(x)$ are a finite set $F$, the integers $Z$, and the rationals $Q$. The first two cases are hardly interesting; $\left(\{0,1\}^{Z}, \sigma\right)$ contains points of all possible orders and the orbit of $\left(x_{n}\right)$ with $x_{n}=0$ iff $n<0$ is homeomorphic to $Z$. Hence from now on we only concentrate on the case $O(x) \approx Q$. As in [A], a homeomorphism $h: Q \rightarrow Q$ is called universally transitive if $Q=\left\{h^{n}(0) \mid n \in Z\right\}$. Without loss of generality we may assume that $h$ is positively recurrent; i.e. $\left\{n \mid h^{n}(0) \in V, n \in N\right\}$ is unbounded for every open set $V$ in $Q$. For convenience we assume that $h$ is negatively recurrent as well. This is not a necessary condition, but it smooths the proof of the theorem. The nonnegatively recurrent case is left to the reader.

Before we turn to the proof let us take a closer look at $\left(\{0,1\}^{Z}, \sigma\right)$. The clopen sets $A=\left\{\left(x_{n}\right)_{n} \mid x_{0}=0\right\}$ has the property that for every two different $x, y \in\{0,1\}^{Z}$, there is an integer $n$ such that $\sigma^{n}(x) \in A$ and $\sigma^{n}(y) \notin A$, or vice versa. We now want to find such a clopen set in $Q$. The first thing that we consider is a neighborhood basis of clopen sets $\mathscr{B}=\left\{B_{n} \mid n \in N\right\}$.

Received by the editors April 8, 1989.

1980 Mathematics Subject Classification (1985 Revision). Primary 54H20. 
It is not hard to find a subset $\left\{U_{i} \mid i \in N\right\} \subset \mathscr{B}$ and integers $n(i)$ such that all $h^{n(i)}\left(U_{i}\right)$ are disjoint, and $A=\bigcup\left\{h^{n(i)}\left(U_{i}\right) \mid i \in N\right\}$ is a clopen subset of $Q$. If we use this set to define $j: Q \rightarrow\{0,1\}^{Z}$ by $j(q)=\left(1_{A}\left(h^{n}(q)\right)\right)_{n}$, then this is a continuous 1-1 map such that $\sigma \circ j=j \circ h$. However $j$ might not be an embedding. So we have to define the clopen set $A$ more carefully. In particular, it has to be a generator. This can be done with the use of the following elementary lemma.

Lemma. Let $h: Q \rightarrow Q$ be a universally transitive homeomorphism, and let $U \subset Q$ be a clopen subset. For $x \in U$ define $n(x)=\min \left\{m \in Z^{+} \mid h^{m}(x) \in U\right\}$. The return map $h_{U}: U \rightarrow U$ defined by $h_{U}(x)=h^{n(x)}(x)$ is a homeomorphism.

Note that the return map is not a homeomorphism but only an embedding if $h$ is not negatively recurrent. This is not a serious obstruction to the proof below.

Proof of the theorem. It suffices to construct a clopen subset $A$ of $Q$ such that $j: Q \rightarrow\{0,1\}^{Z}$ defined by $j(q)=\left(1_{A}\left(h^{n}(q)\right)\right)_{n}$ is an embedding. Let $\mathscr{B}=\left\{B_{n} \mid n \in N\right\}$ be a neighborhood base of 0 such that all $B_{n}$ are clopen. Choose $U_{0} \in \mathscr{B}$ such that $h^{-1}\left(U_{0}\right), U_{0}$, and $h\left(U_{0}\right)$ are pairwise disjoint.

We repeat this construction. Consider the return map $h_{0}$ on $h\left(U_{0}\right)$. Choose a clopen neighborhood $U_{1}$ of $h(0)$ such that $\left(h_{0}\right)^{-1}\left(U_{1}\right), U_{1}$, and $h_{0}\left(U_{1}\right)$ are pairwise disjoint. We can choose $U_{1}$ in such a way that $h^{-1}\left(U_{1}\right)$ is an element of the base $\mathscr{B}$.

We proceed inductively and construct a sequence $U_{0}, U_{1}, \ldots, U_{i}, \ldots$ of disjoint clopen sets. Let $h_{n}: h_{n-1}\left(U_{n}\right) \rightarrow h_{n-1}\left(U_{n}\right)$ be the return map on $h_{n-1}\left(U_{n}\right)$. We can choose a clopen set $U_{n+1}$ such that $\left(h_{n}\right)^{-1}\left(U_{n+1}\right), U_{n+1}$, and $h_{n}\left(U_{n+1}\right)$ are pairwise disjoint and $U_{n+1}$ contains $h_{n}\left(h_{n-1}(\cdots(h(0)) \cdots)\right)$. Without loss of generality we may assume that there are integers $n(i)$ such that $U_{i}=h^{n(i)}\left(B_{i}\right)$ for every $B_{i} \in \mathscr{B}$. One might argue that $A=\bigcup\left\{U_{i} \mid i \in N\right\}$ is
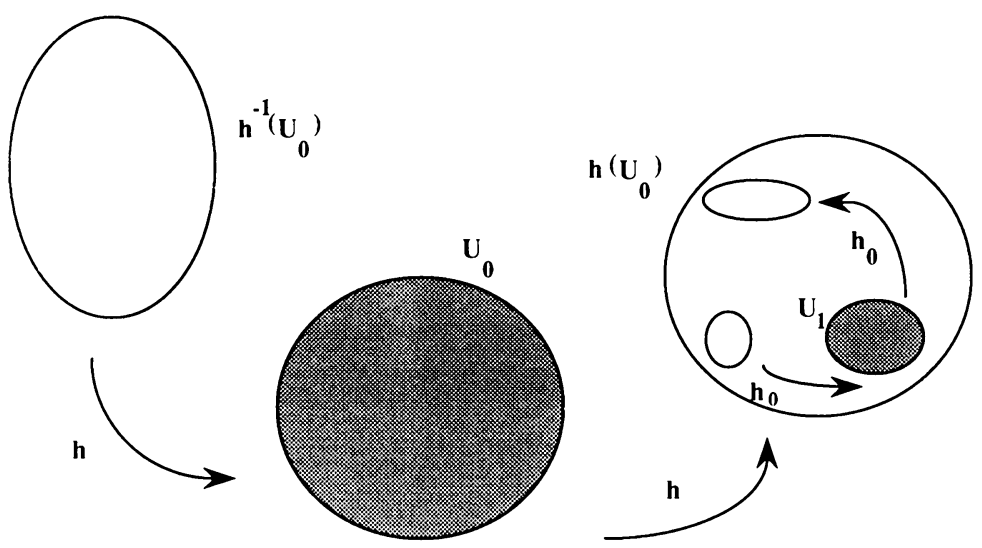
not closed, but this problem can be avoided easily since $Q$ is countable. For instance, let $d$ be a metric on $Q$. It is possible to choose the $U_{n}$ such that $d\left(h^{i}(0), U_{i}\right) \geq \varepsilon_{i}>0$ for all $-n<i<n$ and a suitable sequence $\left(\varepsilon_{i}\right)_{i}$ of positive real numbers. This ensures that $A$ is a clopen subset of $Q$, and therefore, $j$ is continuous. In order to show that $j$, defined by $j(q)=\left(1_{A}\left(h^{n}(q)\right)\right)_{n}$, is in fact an embedding, it suffices to prove that $j\left(B_{i}\right)$ is open in $j(Q)$ for every $i \in N$. Observe that if $x \in j(Q)$, then $x \in j\left(B_{0}\right)=j\left(U_{0}\right)$ iff $x_{-1}=0$ and $x_{0}=1$. Hence $j\left(B_{0}\right)$ is open in $j(Q)$. It is a little more difficult to describe $j\left(B_{1}\right)$. If $q \in B_{1}$, then the first element of the sequence $h^{-1}(q)$, $h^{-2}(q), \ldots, h^{-i}(q), \ldots$ that is contained in $A$ is in fact contained in $B_{0}$. Therefore the first $(j(q))_{n}$ equal to 1 with $n<0$ is a "single" 1 . The set $j\left(B_{1}\right)$ can be described as

$\left\{x \in j(Q) \mid x_{0}=x_{1}=1 \wedge x_{-1}=0 \wedge x_{m-1}=0\right.$ if $\left.m=\max \left\{n \mid n<0 \wedge x_{n}=1\right\}\right\}$, from which, it easily follows that $j\left(B_{1}\right)$ is open in $j(Q)$. It is possible to give a similar description for general $j\left(B_{i}\right)$ to show that it is an open set in $j(Q)$. We conclude that $j$ is an embedding that conjugates $h$ and $\sigma$.

In $[A]$ it is proved that a nonlocally compact orbit in a flow is homeomorphic to the suspension $\Sigma(Q, h)$ for some universally transitive homeomorphism $h$. Therefore we have the following corollary:

Corollary. $\Sigma\left(\{0,1\}^{Z}, \sigma\right)$ contains orbits of every possible topological type.

A question that arises naturally is whether the following holds: let $f: X \rightarrow$ $X$ be a continuous map. Is there an embedding $j_{x}: O^{+}(x) \rightarrow\{0,1\}^{Z}$ that conjugates $f$ and $\sigma$ for every $x \in X$ ? Here $O^{+}(x)$ is the forward orbit of $x$.

The answer to the question is yes, and the proof can be carried out almost exactly like the proof above. Another question one could ask is whether the theorem also holds for homeomorphisms on the irrationals $P$. Of course a restriction has to be put on the homeomorphisms, since both the identity and the shift $\sigma_{P}: N^{Z} \rightarrow N^{Z}$ defined by $\sigma_{P}\left(\left(m_{n}\right)_{n}\right)=\left(m_{n+1}\right)_{n}$ cannot be conjugated to a subsystem of $\left(\{0,1\}^{Z}, \sigma\right)$.

Question. Let $h: P \rightarrow P$ be a minimal homeomorphism. Is there an embedding $j: P \rightarrow\{0,1\}^{Z}$ such that $j \circ h=\sigma \circ j$ ?

\section{REFERENCES}

[A] J. M. Aarts, The structure of orbits in dynamical systems, Fund. Math. 129 (1987), 39-58.

[An] R. D. Anderson, On raising flows and mappings, Bull. Amer. Math. Soc. 69 (1963), 259-264.

[F] H. Furstenberg, Recurrence in ergodic theory and combinatorial number theory, Princeton Univ. Press, Princeton, NJ, 1981.

[MH] M. Morse and G. A. Hedlund, Symbolic dynamics, Amer. J. Math. 60 (1938), 815-866.

Faculteit der Technische Wiskunde en Informatica, Technische Universiteit Te Delft, Vakgroep AW, P. O. Box 356, 2600 AJ Delft, The Netherlands 